\title{
ESTRUCTURACIÓN DE UN PORTAFOLIO DE INVERSIONES CON ACCIONES COLOMBIANAS*
}

\author{
Recibido: 16 de enero de 2016 - Aprobado: 15 de marzo de 2018 \\ DOI: $10.22395 /$ seec.v21n46a7 \\ Luis David Delgado Vélez** \\ María Patricia Durango Gutiérrez ${ }^{* * *}$
}

\section{RESUMEN}

El objetivo de este artículo es evaluar la estructuración de un portafolio con acciones de tres fondos de inversión colectiva del mercado colombiano, basados en el rendimiento del índice Modigliani y Modigliani (M2). La metodología utilizada es un modelo de optimización, cuya función objetivo es minimizar la varianza del portafolio conformado por los tres fondos hasta que se iguale con la varianza del mercado (índice Colcap). Los resultados permiten inferir que la optimización mejoraría el rendimiento de las acciones del fondo Indeacción, al pasar de 7,73\% a 9,86\% efectivo anual; mientras que las del fondo Fiduacción aumentarían su rendimiento de 5,58\% a 27,33\% efectivo anual. Se concluye que, ante el mayor nivel de incertidumbre en la economía y las particularidades del mercado colombiano, es relevante que los administradores de portafolios incluyan en sus análisis algunos performances para mejorar sus decisiones.

\section{PALABRAS CLAVE}

Selección de portafolios; decisiones de inversión; fondos de inversión colectiva; medidas de rentabilidad; Colombia.

\section{CLASIFICACIÓN JEL}

G1 1

\section{CONTENIDO}

Introducción; 1. ¿Qué son los portafolios de inversión?; 2. Los performances de los portafolios de inversión; 3. Los fondos de inversión colectiva; 4. Metodología; 5 . Resultados y discusión; 6. Conclusiones; Bibliografía.

\footnotetext{
Este artículo es resultado de investigación y deviene del proyecto "Observatorio Financiero", adscrito al Grupo de Investigación en Dirección de Empresas - GIDE (clasificado en categoría C por Colciencias en 2017), financiado por la Institución Universitaria Esumer y realizado entre junio y diciembre de 2016.

** Ingeniero industrial, Universidad de Antioquia (Medellín, Colombia). Especialista en ingeniería financiera, Universidad Nacional de Colombia, Sede Medellín. Magíster en finanzas, Universidad Pablo de Olavide, Sevilla, España. Docente investigador, Institución Universitaria Esumer, Medellín, Colombia. Integrante del Grupo de Investigación en Dirección de Empresas de Esumer. Dirección: calle 6 n. 80-126, Medellín, Colombia. Teléfono: 40381 30, Ext. 4116. Correo electrónico: luis.delgado@esumer.edu.co

** Administradora de negocios, especialista en finanzas y magíster en finanzas, Universidad Eafit, Medellín Colombia). Docente investigadora, Institución Universitaria Esumer, Medellín, Colombia. Integrante del Grupo de Investigación en Dirección de Empresas de Esumer. Dirección: calle 6 n. . 80-126, Medellín, Colombia. Teléfono: 40381 30, Ext. 4121. Correo electrónico: maria.durango@esumer.edu.co
} 


\title{
STRUCTURING OF AN INVESTMENT PORTFOLIO WITH COLOMBIAN SHARES
}

\begin{abstract}
The main goal of this paper is to evaluate the structuring of a portfolio with shares of three collective investment funds of the Colombian market, based on the performance of the Modigliani and Modigliani index (M2). The methodology used is an optimization model, whose objective function is to minimize the variance of the aforementioned portfolio until it is equal to the market variance (Colcap index). The results allow to infer that optimization would improve the performance of the Indeacción fund shares, going from $7.73 \%$ to $9.86 \%$ annual cash; while those of the Fiduacción fund would increase their performance from $5.58 \%$ to $27.33 \%$ annual cash. It is concluded that, given the greater level of uncertainty regarding the economy and the particularities of the Colombian market, it is important that portfolio managers include in their analysis some performances to improve their decisions.
\end{abstract}

\section{KEYWORDS}

Portfolio selection; investment decisions; collective investment funds; profitability measures; Colombia.

\section{JEL CLASSIFICATION}

G11

\section{CONTENT}

Introduction; 1. What are the Investment Portfolios?; 2. The Performances of Investment Portfolios; 3. Collective Investment Funds; 4. Methodology; 5. Results and Discussion; 6. Conclusions; Bibliography.

\section{ESTRUTURAÇÃO DE UM PORTFÓLIO DE INVESTIMENTOS COM AÇÕES COLOMBIANAS}

\section{RESUMO}

O objetivo deste artigo é avaliar a estruturação de um portfólio com ações de três fundos de investimento coletivo do mercado colombiano, baseados no rendimento do índice Modigliani e Modigliani (M2). A metodologia utilizada é um modelo de otimização, cuja função principal é minimizar a variância do portfólio formado pelos três fundos até que se iguale à variância do mercado (índice Colcap). Os resultados permitem inferir que a otimização melhoraria o rendimento das ações do fundo "Indeacción" ao passar de 7,73\% para 9,86\% efetivo anual; enquanto as ações do fundo "Fiduacción" aumentariam seu rendimento de 5,58\% para 27,33\% efetivo anual. Conclui-se que, frente ao maior nível de incerteza na economia e às particularidades do mercado colombiano, é relevante que os administradores de portfólios incluam algumas performances em suas análises para melhorar suas decisões.

\section{PALAVRAS-CHAVE}

Seleção de portfólios; decisões de investimento; fundos de investimento coletivo; medidas de rentabilidade; Colômbia.

\section{CLASSIFICAÇÃO JEL}

G11

\section{CONTEÚDO}

Introdução; 1 . O que são os portfólios de investimento?; 2. As performances dos portfólios de investimento; 3 . Os fundos de investimento coletivo; 4. Metodologia; 5. Resultados e discussão; 6. Conclusões; Bibliografia. 


\section{INTRODUCCIÓN}

Para el caso colombiano son varios los eventos que han impactado el desarrollo del mercado accionario. Inicialmente, y como consecuencia de la crisis financiera mundial de 2008, la quiebra de Lehman Brothers y el rescate de American Inter Group por parte de la Reserva Federal estadounidense afectaron el normal funcionamiento de los mercados financieros y generaron una reducción importante en la liquidez global, incluido el mercado colombiano, cuyo índice alcanzó una desvalorización de 14,02\%. Otro hito importante ocurrió en 2012, como lo afirman Gómez y Melo (2014), con la suspensión temporal de la acción de Interbolsa' y su posterior liquidación forzosa, que ocasionó gran desconfianza entre los agentes del mercado y, en efecto, una desvalorización generalizada en las 18 acciones más importantes del mercado. Seguidamente, el Banco de la República (2014) afirmó que la disminución aproximada de un $40 \%$ en los precios internacionales del petróleo, acaecida en el segundo semestre de 2014, también trajo consecuencias nefastas para las acciones colombianas, con una desvalorización del 5,8\% en el índice Colcap y, específicamente, una caída superior al 50\% en la acción de Ecopetrol, siendo esta última la más ponderada y con el mayor número de personas naturales inversionistas.

De otro lado, los expertos de la industria financiera se han limitado a medir los rendimientos de sus portafolios basados casi por completo en el rendimiento total; ello representa una medida incompleta del desempeño de una cartera, ya que se ignora el riesgo. En este sentido, Mir y Olmeda (1999) afirman que una de las prácticas más comunes para evaluar el comportamiento de un portafolio de inversión es comparar su rendimiento frente a carteras no gestionadas o un índice del mercado; si el rendimiento es superior, se concluye que ha sido una gestión eficiente de su administrador. No obstante, el análisis es incompleto si se excluye el nivel de exposición al riesgo. Al margen, Modigliani y Modigliani (1997) afirman que, ante la mayor ocurrencia de eventos extremos en los mercados financieros en los últimos 20 años, se ha hecho necesario incrementar el uso del performance para aumentar los rendimientos esperados, al aceptar un mayor nivel de riesgo o incertidumbre en el rango de posibles resultados.

Otro factor importante, que se constituye en un factor de riesgo adicional para los administradores de portafolios, es la estructura y composición del mercado accionario colombiano. En este sentido, Uribe y Ulloa (2011), al igual que Uribe y Fernández (2014), afirman que la alta concentración accionaria en unos pocos conglomerados, las considerables dependencias de algunos títulos entre sí, el excesivo

Una de las firmas comisionistas de mayor tamaño, principal comprador de deuda pública y proveedora importante de liquidez en el mercado financiero colombiano. 
protagonismo de inversionistas institucionales como los fondos de pensiones (Pino, Uribe y Jiménez, 2017) y capitales foráneos, la falta de liquidez, y la presencia de empresas del gobierno que representan ciertas garantías implícitas, dejan en evidencia la vulnerabilidad del mercado, facilitan la generación de burbujas especulativas que pueden ir en contravía de los intereses de pequeños inversionistas, y dejan pocas posibilidades para la diversificación del riesgo.

Por todo lo anterior, es necesario incorporar otras medidas alternativas en la gestión de los portafolios, que ayuden a tomar decisiones más favorables para los inversionistas en la combinación ideal de liquidez - riesgo - rentabilidad. En consecuencia, el objetivo de este artículo es evaluar la estructuración de un portafolio en el que se incluyen acciones de tres fondos de inversión colectiva (FIC) del mercado de valores colombiano, en el cual se utiliza como factor de referencia el performance del índice M2, para mejorar el rendimiento de sus portafolios al modificar sus posiciones de riesgos, con base en un índice de referencia.

En el desarrollo de este artículo se plantean, en primer término, los aspectos teóricos más relevantes de la teoría de los portafolios de inversión, con énfasis en el índice Modigliani y Modigliani (M2), además de describir las particularidades de los fondos de inversión colectiva en Colombia. Seguidamente, en la metodología, se plantea el tipo de investigación y la manera como se planteó la estrategia para aplicar este índice al mercado accionario colombiano, basado en tres FIC y el índice Colcap, en un horizonte temporal de seis meses. Posteriormente, en los resultados, se explica cómo mejoraría el rendimiento de las carteras, a partir de un modelo de optimización que permite ajustar los riesgos al igualarlos con el del mercado; y las conclusiones permiten inferir, entre otras cosas, que las particularidades del mercado accionario colombiano no dan mucho margen para componer portafolios en acciones diversificados.

\section{1. ¿QUÉ SON LOS PORTAFOLIOS DE INVERSIÓN?}

Para estructurar un portafolio o cartera de inversión, se debe encontrar una composición óptima de títulos que entreguen el menor riesgo para un máximo retorno. Para lograrlo, el inversionista debe identificar, primero, cuáles son los títulos que va a comprar; y segundo, se debe definir cuánto se va a invertir en cada título. La elección de los activos financieros depende de tres características esenciales: liquidez, rentabilidad y riesgo. Usualmente, los activos más líquidos son aquellos con los que se realiza un mayor número y volumen de transacciones en los mercados más organizados y desarrollados. Respecto a la rentabilidad, Escobar y Cuartas (2006) afirman que esta hace referencia a los beneficios que se obtienen de una inversión 
o una empresa; según la Real Academia Española, "el riesgo es la contingencia o proximidad de un daño"; y matemáticamente se define como la desviación estándar o la volatilidad. Como lo indica García (2013), gestionar los portafolios de inversión es una parte de la administración de inversiones que comprende las siguientes fases:

i. Analizar los títulos a través de la rentabilidad esperada, la volatilidad y la covarianza.

ii. Analizar las carteras desde sus rendimientos y riesgos probables, así como el monto invertido.

iii. Entre las carteras seleccionadas, encontrar la óptima de acuerdo con las preferencias y objetivos de los inversionistas.

De otro lado, Crespo y Fernández (2008, p. 67) afirman que, cuando las cotizaciones corresponden a pequeños periodos de tiempo, como en el caso de las acciones cuyos precios se suministran diariamente, existe una manera más aproximada de calcular la rentabilidad real, especialmente mediante el cálculo continuo o logarítmico de la relación de las cotizaciones de los activos o rendimiento continúo, como se plantea en la ecuación (1).

Donde:

$$
\mathrm{R}_{t}=\ln \left(\frac{\mathrm{P}_{t}}{\mathrm{P}_{t-1}}\right)
$$

$R_{t}=$ Rentabilidad del título

$P_{t}=$ Cotización del activo financiero al final del período

$P_{t-1}=$ Cotización del activo financiero al inicio del período

El rendimiento esperado del portafolio se define como la suma - producto de las rentabilidades individuales de los títulos por sus respectivos pesos o ponderaciones de cada activo, como se plantea en la ecuación (2).

$$
r_{p}=\sum_{i=1}^{\mathrm{N}} \mathrm{X}_{i} r_{i}
$$

Donde:

$r_{p}=$ Rendimiento esperado del portafolio

$\mathrm{X}_{\mathrm{i}}=$ Proporción del valor inicial del portafolio invertido en el título i

$r_{\mathrm{i}}=$ Rendimiento esperado del título $\mathrm{i}$ 
La volatilidad hace referencia a la diferencia que podría existir entre el rendimiento real y el esperado por el inversionista. La ecuación (3) ilustra el cálculo de la volatilidad individual o riesgo del título.

Donde:

$$
\sigma=\sqrt{\left[\frac{1}{n-1} * \sum_{i=1}^{n}\left(r_{i}-r\right)^{2}\right]}
$$

$\sigma=$ Desviación estándar o volatilidad del título

$\mathrm{R}=$ Rendimento esperado del título i

$\mathrm{N}=$ Número de sesiones en un año. Para datos diarios, $\mathrm{N}=252$.

En cuanto al riesgo del portafolio, Gordon, Sharpe y Bailey (2003) afirman que este se calcula a partir de las volatilidades individuales de los títulos, sus covarianzas y las ponderaciones de los mismos, y considera la forma en que varían los rendimientos entre sí, como lo muestra la ecuación (4).

$$
\sigma_{p}=\left[\sum_{i=1}^{n} \sum_{i=1}^{n} \mathrm{X}_{i} \mathrm{X}_{j} \sigma_{i j}\right]
$$

Donde:

$\sigma_{p}=$ Riesgo del portafolio

$\sigma_{i j}=$ Covarianza de los rendimientos entre el valor i y el valor $\mathrm{j}$.

El tercer aspecto señalado por García (2013) tiene como finalidad determinar un conjunto de carteras posibles -llamadas carteras eficientes- que proporcionan la máxima rentabilidad para un nivel de riesgo dado, y de estas se selecciona la óptima. Este proceso comprende dos estados: el primero comienza con la observación y experiencia del administrador del portafolio, y finaliza con las expectativas de rentabilidad de los títulos disponibles en el mercado; y el segundo permite la selección de los títulos. Markowitz (1952) define este último como el modelo de media-varianza, dado que se basa en la esperanza matemática y la desviación típica de la variable aleatoria de la rentabilidad. Para su comprensión es necesario considerar los siguientes supuestos:

1. La amplitud del horizonte temporal de la inversión es única.

2. La rentabilidad de los activos es una variable aleatoria con una distribución de probabilidad conocida. 
3. La media y la desviación típica de la variable aleatoria de la rentabilidad constituyen el rendimiento esperado y el riesgo que asume el inversionista.

4. Las decisiones de los inversionistas están en función del riesgo y la rentabilidad.

5. Los inversionistas tienen un comportamiento racional.

6. No hay impuestos ni inflación. No se consideran los costos de transacción.

7. Es posible comprar cualquier fracción del activo.

En términos formales, el problema se reduce a la expresión matemática que se presenta en la ecuación (5), que garantiza la maximización de la rentabilidad.

$$
\begin{gathered}
\text { Máx Rentab }=\sum_{i=1}^{n} \sum_{i=1}^{n} \mathrm{X}_{i} \mathrm{X}_{j} \sigma_{i j} \\
\text { S.a. } \mathrm{E}_{c}=\sum_{i}^{\mathrm{N}} \mathrm{X}_{i} \mathrm{E}_{i} \\
\mathrm{X}_{1}+\ldots+\mathrm{X}_{\mathrm{N}}=1 \\
\mathrm{X}_{1}+\ldots+\mathrm{X}_{\mathrm{N}} \geq 0
\end{gathered}
$$

Donde:

$$
\begin{aligned}
& \mathrm{Xi}=\text { Peso de cada valor en la cartera } \mathrm{C} \\
& \mathrm{Ei}=\text { Esperanza del rendimiento del título i o de la cartera }
\end{aligned}
$$

\section{EL PERFORMANCE DE LOS PORTAFOLIOS DE INVERSIÓN}

De manera complementaria, la revisión teórica sobre administración de portafolios de inversión remite inicialmente a Markowitz (1952), a través del modelo de media-varianza. Posteriormente, otros autores desarrollaron una serie de indicadores en la creciente industria de los portafolios de inversión, llamadas medidas de performance. Moreno y Olmeda (2003) los definen como una operación matemática que permiten valorar los resultados de los activos financieros en términos de la relación riesgo - rentabilidad. A partir del modelo de Markowitz, Sharpe (1966) propuso una nueva medida para valorar el desempeño de los portafolios, calculada como la diferencia entre la rentabilidad anualizada y la tasa libre de riesgo, dividida entre el riesgo. Treynor (1966), por su parte, definió su ratio con base en el modelo inicial de Sharpe, pero consideró solo el riesgo sistemático, dado que lo considera como el único relevante que se debe diversificar. Jensen (1968), formuló el Alpha 
de Jensen, que mide la rentabilidad de un portafolio por encima del mercado (sus competidores) y se calcula como el diferencial de rentabilidad real, ajustado por el riesgo sistemático. Estas son, entre otras, las medidas más tradicionales.

El índice M2, por su parte, fue una medida desarrollada en 1997 por Francis y Leah Modigliani que permite medir el rendimiento de la inversión ajustada al riesgo, o Risk-Adjusted-Performance (RAP). Este último iguala el riesgo del portafolio al del mercado o hace benchmark con el riesgo de un índice de referencia, a través de un proceso de apalancamiento (o desapalancamiento), como lo muestra el gráfico 1, a través de la combinación del portafolio inicial con un activo libre de riesgo $R_{f}$, hasta alcanzar el nivel deseado, lo que también variaría la rentabilidad.

\section{Gráfico 1. M2 ajustado por apalancamiento}

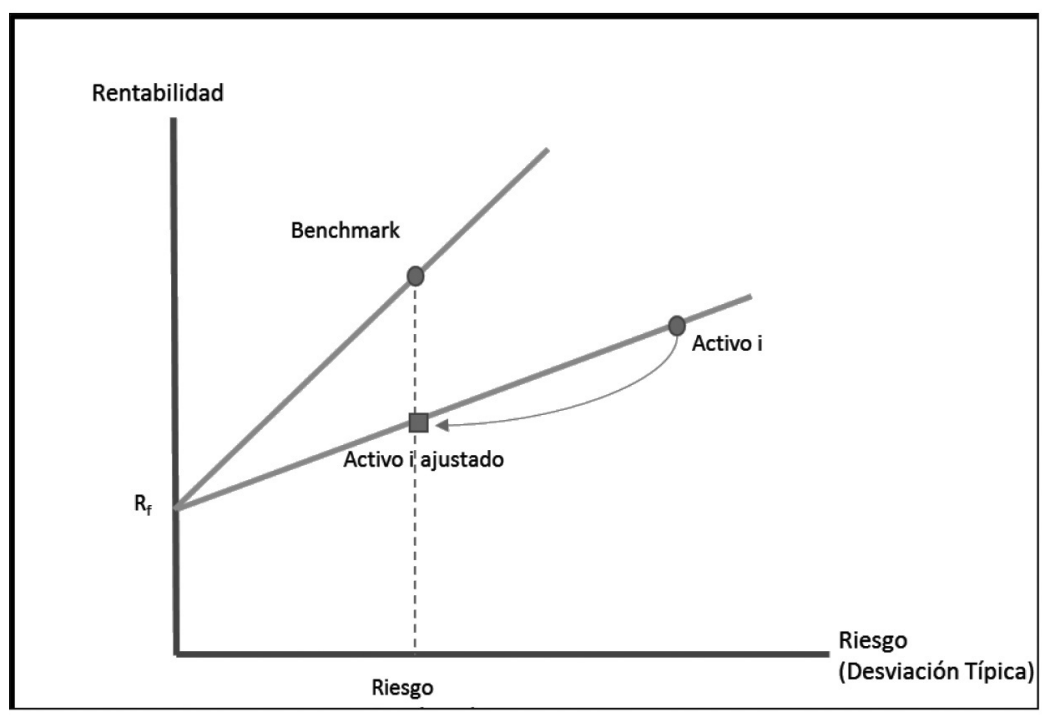

Fuente: Moreno y Olmeda (2003, p. 14).

El gráfico 1 muestra la relación entre el riesgo (eje horizontal) y la rentabilidad (eje vertical), como lo afirma Winston (2011), quien explica que este procedimiento consiste en comparar un portafolio más riesgoso que el índice (línea del Activo i) y se ajusta hasta igualarlo (línea Benchmark), al agregar una cantidad de efectivo "c" que permite reducir la variabilidad del portafolio; de esta manera, el RAP es el rendimiento esperado del portafolio combinado. De manera inversa, si el objetivo es aumentar el riesgo del portafolio para igualarlo al índice, se resta la cantidad de efectivo "c", para aumentar su variabilidad hasta que calce con el índice, como lo indica la ecuación (6). 


$$
\mathrm{RAP}=\frac{\sigma_{b}}{\sigma_{i}}\left(\mathrm{R}_{i}-\mathrm{R}_{f}\right)+\mathrm{R}_{f}
$$

Donde:

$\sigma_{6}=$ Desviación del índice (benchmark)

$\sigma_{i}=$ Desviación de la cartera i

$\mathrm{R}_{i}=$ Rentabilidad de la cartera $\mathrm{i}$

$\mathrm{R}_{f}=$ Tasa libre de riesgo

Esta igualdad permite expresar un diferencial de rentabilidad (medido en puntos básicos) para un mismo nivel de riesgo. No obstante, Zhiwu y Peter (1996) afirman que el modelo exige coincidencia entre la fecha de maduración del activo libre de riesgo y la fecha de valoración para evitar sesgos.

\section{LOS FONDOS DE INVERSIÓN COLECTIVA}

Los FIC existentes en el mercado de valores colombiano son equivalentes a los fondos mutuos en los mercados internacionales, y captan dinero del público para invertir en activos financieros y no financieros a través de expertos que estructuran portafolios con base en reglamentos, políticas de inversión y el perfil de riesgo de los inversionistas. La misma ley determina que las instituciones financieras habilitadas para estructurar los FIC son Sociedades Fiduciarias, firmas comisionistas de bolsa y sociedades administradoras de inversión, que ofrecen carteras abiertas o cerradas, según la obligación de redimir las inversiones en cualquier momento o al vencimiento del plazo pactado.

Los FIC tienen como finalidades, entre otras, fomentar la cultura del ahorro en la población; diversificar las inversiones al tener la posibilidad de acceder a otros mercados de manera colectiva; y delegar las decisiones de inversión en personas expertas, formadas profesionalmente en la teoría financiera, que buscan el equilibrio entre la liquidez, el riesgo y la rentabilidad. En el curso de esta búsqueda, y con el propósito de alcanzar el mayor grado de transparencia y comunicación efectiva con los clientes, el artículo 3.1.1.9.8 del Decreto 1242 de 2013, promulgado por el Ministerio de Hacienda y Crédito Público, exige a los FIC la publicación de sus fichas técnicas, para que sus inversionistas se documenten sobre las características de la inversión en términos de la valoración de la unidad, número de unidades que la componen, tipo de cartera y condiciones de la inversión (retiros, adiciones, comisiones, remuneraciones por éxito, sanciones y gastos, entre otros). La ficha 
técnica también describe las características del portafolio en términos de su composición (calificación, tipo de renta, emisor, sector económico y plazos, entre otros), además del comportamiento histórico (rentabilidad y volatilidad) y una descripción de las estrategias de inversión, conforme a unas políticas previamente establecidas. Al margen de lo anterior, el administrador del portafolio está en la obligación de emitir un informe de desempeño que muestre los resultados obtenidos para un periodo a través de los estados contables.

\section{METODOLOGÍA}

Se estructuró un portafolio conformado por tres de los FIC más representativos en acciones del mercado colombiano (Indeacción, Fiduacción y Daviplus), con las cotizaciones del valor diario de sus unidades en el primer semestre de 2015. Para el mismo periodo también se tomaron las cotizaciones históricas del índice Colcap y los TES a 10 años. Con estas series de datos se procedió a calcular las rentabilidades continuas diarias y sus respectivos riesgos. Seguidamente, se calculó el rendimiento combinado diario ajustado de cada portafolio, como se ilustra en la ecuación (7).

$$
\mathrm{R}_{\text {dajustado }}=\frac{\left(1+\mathrm{R}_{p}\right)+\mathrm{C}\left(1+\mathrm{R}_{f}\right)}{1+\mathrm{C}}-1
$$

Donde:

$\mathrm{R}_{p}=$ Rentabilidad diaria de cada portafolio

$\mathrm{R}_{f}=$ Tasa libre de riesgo (rentabilidad diaria de los TES a 10 años)

$\mathrm{C}=$ Cantidad de efectivo que se debe adicionar o restar para igualar la varianza de cada portafolio a la del mercado Colcap.

Tras lo anterior, se calcularon la rentabilidad promedio y la desviación de cada portafolio combinado con la cantidad de efectivo "c"; y por último, se calculó el cuadrado de la diferencia entre la desviación de los rendimientos ajustados y la del índice Colcap. Con base en este último cálculo se procedió a definir la función objetivo como la minimización de la suma de los tres resultados anteriores, cuyas celdas cambiantes corresponden a cada una de las cantidades " $c$ " que se deben adicionar o restar. La única restricción del modelo radica en que todos los valores de "c" deben ser mayores o iguales a - 1. Para la solución del modelo se utilizó Solver de Microsoft Excel, que permitió obtener la cantidad de efectivo óptima que cada FIC debería sumar o restar, para igualar la varianza del mercado Colcap y así mejorar la rentabilidad. 


\section{RESULTADOS Y DISCUSIÓN}

Para el análisis se tomó como índice benchmark el Colcap, que sirvió como referencia para comparar los tres FIC nombrados. El Colcap es un índice ponderado por capitalización bursátil de las 20 compañías más líquidas, creado en 2008 y con una base de 1.000 puntos, que se basa en una función de selección compuesta por tres variables: frecuencia, rotación y volumen. Se tomaron los valores históricos comprendidos entre el 1 de enero y el 30 de junio de 2015 y con el propósito de optimi-

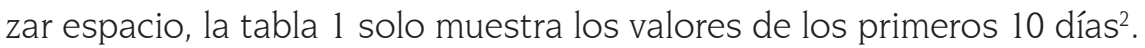

Tabla 1. Cotizaciones históricas del mercado y los tres FIC

\begin{tabular}{|c|c|c|c|c|}
\hline Fecha & Colcap & Indeacción & Fiduacción & Daviplus \\
\hline $1 / 01 / 2015$ & $1.483,89$ & $10.820,75$ & $24.421,53$ & $8.578,13$ \\
\hline $2 / 01 / 2015$ & $1.483,89$ & $10.762,93$ & $24.049,32$ & $8.438,20$ \\
\hline $3 / 01 / 2015$ & $1.483,89$ & $10.763,36$ & $24.047,36$ & $8.437,77$ \\
\hline $4 / 01 / 2015$ & $1.483,89$ & $10.763,79$ & $24.045,40$ & $8.437,34$ \\
\hline $5 / 01 / 2015$ & $1.418,74$ & $10.605,16$ & $23.052,37$ & $8.106,99$ \\
\hline $6 / 01 / 2015$ & $1.419,92$ & $10.604,42$ & $23.131,84$ & $8.095,76$ \\
\hline $7 / 01 / 2015$ & $1.422,20$ & $10.634,77$ & $23.280,50$ & $8.111,80$ \\
\hline $8 / 01 / 2015$ & $1.433,37$ & $10.667,82$ & $23.430,51$ & $8.187,19$ \\
\hline $9 / 01 / 2015$ & $1.426,77$ & $10.657,29$ & $23.353,50$ & $8.163,56$ \\
\hline $10 / 01 / 2015$ & $1.426,77$ & $10.658,40$ & $23.351,75$ & $8.163,20$ \\
\hline
\end{tabular}

Fuente: Superfinanciera y Bolsa de Valores de Colombia.

Indeacción, Fiduacción y Daviplus son FIC en acciones de tres instituciones financieras de Colombia. La Fiduciaria Bancolombia señala que Indeacción, su fondo, está diseñado para brindar a los inversionistas una rentabilidad asociada al desempeño del índice accionario Colcap, con retornos de mediano y largo plazo. Para la Fiduciaria Fidubogotá, por su parte, la estrategia de su fondo, Fiduacción, es mantener inversiones en acciones de acuerdo con el comportamiento esperado; y su objetivo es ofrecer un portafolio diversificado con mayor relación retorno/ riesgo, además de un mejor comportamiento que los índices accionarios locales y

\footnotetext{
${ }^{2}$ La información completa utilizada en este ejercicio de optimización de portafolios puede ser solicitada a los autores vía correo electrónico (a las direcciones consignadas anteriormente).
} 
los fondos de renta variable de la competencia. La Fiduciaria Davivienda, a su turno, afirma que la política de su fondo, Daviplus, se basa en inversiones de largo plazo, en acciones locales, cuyos rendimientos tienen altas volatilidades.

Al compararse con otros mercados mundiales, una de las limitaciones del mercado accionario colombiano radica en el bajo volumen transado y su poca profundidad, lo que permite observar en este caso composiciones muy similares en los tres portafolios al invertir en las mismas compañías -Bancolombia, Grupo Sura, Ecopetrol, Grupo Argos, Nutresa y Cementos Argos-, como lo muestra el gráfico 2. Estas seis compañías concentran el 92\% de Indeacción, el 62\% de Fiduacción y el $58 \%$ de Daviplus.

\section{Gráfico 2. Composición de los FIC}

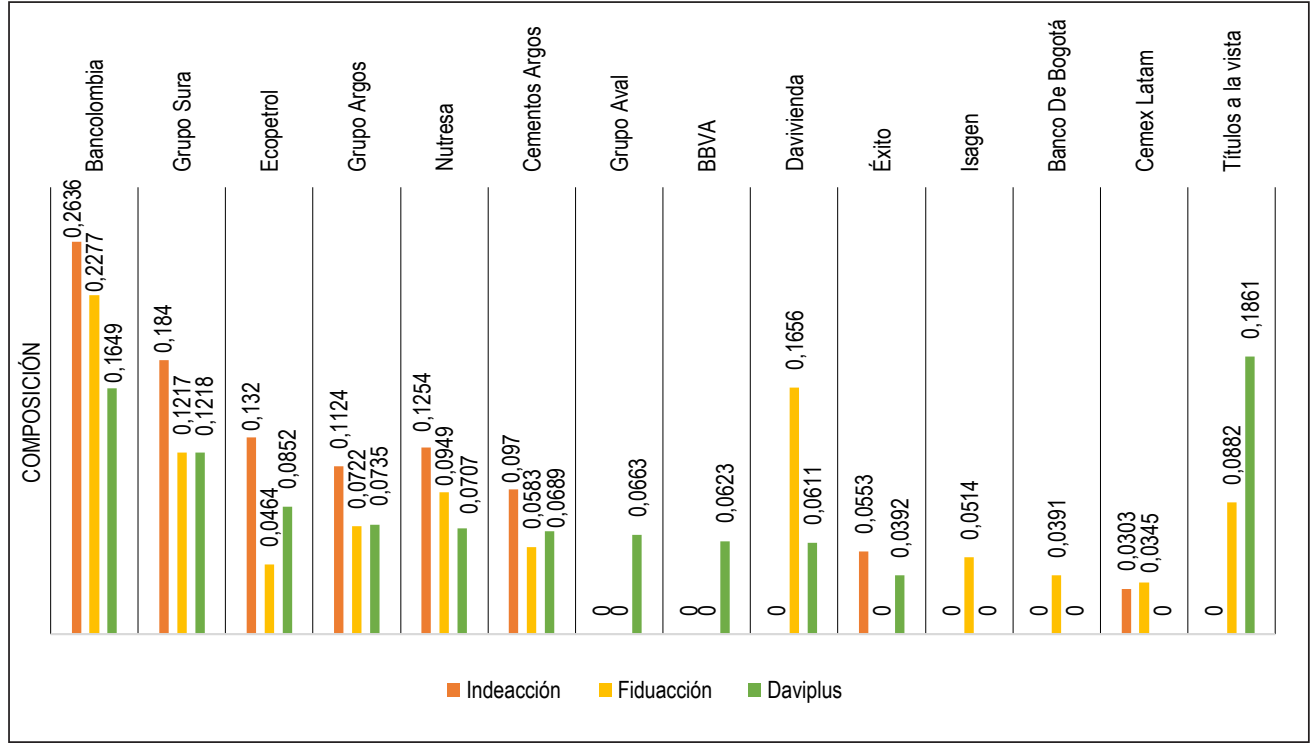

Fuente: elaboración propia.

Al analizar la composición por la actividad económica, la ilustración anterior indica que los portafolios tienen importantes concentraciones en compañías del mismo sector financiero, tales como Bancolombia, Grupo Sura, Grupo Aval, BBVA, Davivienda y Banco de Bogotá, además de diversificarse en otros sectores como cemento, petróleo, energía y alimentos. El cálculo promedio de las rentabilidades y desviaciones anuales muestran a Daviplus como la de mayor relación riesgo-rentabilidad, con el $11,26 \%$ y $12,74 \%$ respectivamente; es el único portafolio que supera al mercado con más rentabilidad y menos riesgo, como lo muestra a tabla 2. 
Estructuración de un portafolio de inversiones con acciones colombianas

Tabla 2. Rentabilidades y desviaciones promedio de los FIC y el índice Colcap

\begin{tabular}{|r|c|c|c|c|}
\hline Estadístico & Colcap & Indeacción & Fiduacción & Daviplus \\
\hline Desviación estándar & $14,02 \%$ & $4,17 \%$ & $1,84 \%$ & $11,26 \%$ \\
\hline Rentabilidad media & $10,63 \%$ & $7,73 \%$ & $5,58 \%$ & $12,74 \%$ \\
\hline
\end{tabular}

Fuente: elaboración propia con datos de Superfinanciera y BVC.

Al obtener volatilidades inferiores al mercado para los tres portafolios, fue necesario disminuir una cantidad de efectivo "c" de tal manera que aumente el riesgo de cada uno, hasta igualarlo con el índice Colcap. Inicialmente, se asignó de manera aleatoria un valor de -0,24 a Indeacción, con el fin de obtener el rendimiento combinado diario de cada portafolio $\left(\mathrm{R}_{\text {d ajustado }}\right)$. Luego, se procedió a calcular la rentabilidad ajustada al riesgo con el promedio de las rentabilidades diarias ajustadas, con la ecuación (8) de la RAP del portafolio.

$$
\operatorname{RAP}_{\text {Indeacción }}=\sum^{180} \frac{\left(1+R_{i}\right)+c\left(1+R_{f}\right)}{(}=8,016 \% \mathrm{EA}
$$

Donde:

$\mathrm{R}_{i}=$ Rentabilidad diaria de Indeacción

$\mathrm{R}_{f}=$ Tasa libre de riesgo (rentabilidad diaria de los TES a 10 años)

$\mathrm{C}=$ Cantidad de efectivo que se adicionó o restó a Indeacción para igualar la varianza de cada portafolio a la del mercado Colcap.

El valor obtenido, de 8,016\% efectivo anual para el promedio de la rentabilidad media ajustada, indica que al reducir en 24 centavos por cada peso invertido en el portafolio de Indeacción, el riesgo pasaría de 4,17\% (valor original) a 5,48\%. No obstante, el objetivo es alcanzar el valor de 14,024\% anual, que corresponde al índice benchmark del Colcap. De manera análoga se calculó el RAP para los demás fondos, cuyos rendimientos se detallan en la tabla 3. Sobre el valor de los RAP diarios ajustados se procedió a calcular la desviación estándar, para obtener finalmente el RAP de cada uno de los fondos. 
Luis David Delgado Vélez - María Patricia Durango Gutiérrez

Tabla 3. Valores iniciales de la rentabilidad media y la desviación

\begin{tabular}{|l|c|c|c|c|}
\hline \multicolumn{1}{|c|}{ Estadístico } & Colcap & Indeacción & Fiduacción & Daviplus \\
\hline Desviación estándar & $14,024 \%$ & $5,843 \%$ & $6,088 \%$ & $13,244 \%$ \\
\hline Rentabilidad media & $10,628 \%$ & $8,016 \%$ & $15,302 \%$ & $13,813 \%$ \\
\hline Cantidad de efectivo C & & $-0,2400$ & $-0,3200$ & $-0,1500$ \\
\hline
\end{tabular}

Fuente: elaboración propia.

Para obtener los valores óptimos de "c", se resolvió en Microsoft Excel el siguiente modelo de optimización:

a) Definición de variables

$\mathrm{RAP}_{\text {indeacción }}=$ Risk Adjusted Performance de Indeacción

$\mathrm{RAP}_{\text {Induacción }}=$ Risk Adjusted Performance de Fiduacción

$\mathrm{RAP}_{\text {Daviplus }}=$ Risk Adjusted Performance de Daviplus

colcap $=\quad$ Riesgo del índice Colcap

b) Función objetivo:

$\operatorname{MinZ}:\left(\sigma-\operatorname{RAP}_{\text {Indeación }}\right)^{2}+\left(\sigma-\operatorname{RAP}_{\text {Fiduación }}\right)^{2}+\left(\sigma-\operatorname{RAP}_{\text {Daviplus }}\right)^{2}$

c) Restricciones:

Sujeto a:

$\operatorname{RAP}_{\text {Indeación }} \geq-1$

$\operatorname{RAP}_{\text {Fiduación }} \geq-1$

$\operatorname{RAP}_{\text {Daviplus }} \geq-1$

La tabla 4 muestra el resultado óptimo que permite igualar el riesgo de cada uno con el índice Colcap. 
Tabla 4. Resultados de la optimización

\begin{tabular}{|l|c|c|c|c|}
\hline \multicolumn{1}{|c|}{ Estadístico } & Colcap & Indeacción & Fiduacción & Daviplus \\
\hline Desviación estándar & $14,024 \%$ & $14,025 \%$ & $14,024 \%$ & $14,024 \%$ \\
\hline Rentabilidad media & $10,628 \%$ & $9,861 \%$ & $27,333 \%$ & $14,238 \%$ \\
\hline Cantidad de efectivo C & & $-0,7029$ & $-0,7048$ & $-0,1973$ \\
\hline
\end{tabular}

Fuente: elaboración propia.

Al tener un riesgo anual de 14,24\% para el mercado, el modelo óptimo indica que se debe sustraer \$ 0,7029 en efectivo por cada peso invertido en Indeacción para asumir la misma volatilidad; en consecuencia, el rendimiento pasaría de 7,73\% a 9,86\% efectivo anual. Para el caso de Fiduacción se restaría 0,7048 y la rentabilidad mejoraría sustancialmente, al pasar de 5,58\% a 27,33\%; mientras que Daviplus reduciría 0,1972 de efectivo para alcanzar una rentabilidad promedio de 14,23\% efectivo anual.

\section{CONCLUSIONES}

EL mayor nivel de incertidumbre que viven los mercados accionarios en todo el mundo trae consigo mayores exigencias para administrar los portafolios de inversión en acciones con estrategias más fundamentadas que contribuyan a niveles óptimos de liquidez, riesgo y rentabilidad. Por ello, es conveniente que expertos e inversionistas incluyan en sus análisis algunos performances que les ayuden a mejorar las decisiones. En este sentido, el M2 es, entre otras cosas, una medida que permite igualar el riesgo de un portafolio al del mercado, a través de un proceso de apalancamiento (o desapalancamiento), al invertir una cantidad "c" en un activo libre de riesgo.

Los FIC constituyen una buena alternativa para que las personas naturales inversionistas en Colombia dispongan su dinero en el mercado de capitales. A través de estos mecanismos, las personas que cuentan con poco conocimiento sobre la estructura y la dinámica de estos mercados tienen la posibilidad de diversificar el riesgo de sus inversiones, participar con bajos montos y delegar las decisiones de comprar en expertos. No obstante, el tamaño del mercado colombiano, expresado en términos de emisores y volúmenes transados, no brinda mucho margen de maniobra para que los administradores estructuren sus portafolios. En el caso de los FIC en acciones, las composiciones son muy similares en términos de diversificación: las diferencias se marcan principalmente por los montos invertidos en cada activo y los tipos de carteras. 


\section{REFERENCIAS}

Banco de la República (2014). Reporte de estabilidad financiera: marzo de 2014. Bogotá: Banco de la República, 120p.

Crespo, J. y Fernández, C. (2008). Descubriendo el Mercado (I): medidas de posición central. En: Bolsa: revista mensual de bolsas y mercados españoles, n. ${ }^{\circ}$ 178, p. 61-64.

Escobar, H., y Cuartas, V. (2006). Diccionario económico financiero (Tercera ed.). Medellín: Universidad de Medellín, 484p.

García Boza, J. (2013). Inversiones financieras: selección de carteras. Madrid: Pirámide, 385p.

Gómez, J. y Melo, L. (2014). Efectos de ángeles caídos en el mercado accionario colombiano: estudio de eventos del caso Interbolsa. En: Ensayos sobre política económica, Vol. 32, n. 75, marzo-junio, p. 23-27.

Gordon, A.; Sharpe, W. y Bailey, J. (2003). Fundamentos de inversiones. México: Pearson Educación: 687p.

Jensen, M. (1968). The Performance of Mutual Funds in the period 1945-1964. En: Journal of Finance, Vol. 23, n. 2, p. 389-416.

Markowitz, H. (1952). Portfolio Selection. The Journal of finance. En: Journal of Finance, Vol. 7, n. ${ }^{\circ} 1$, p. 77-91.

Mir, F. y Olmeda, I. (1999). Medidas de performance de las bolsas europeas. En: Series de Economía de la empresa, Vol. 1, n. ${ }^{\circ}$ 3, p. 625-631.

Modigliani, F. y Modigliani, L. (1997). Risk-Adjusted Performance. En: The Journal of Portfolio Management Winter, Vol. 23, n.ํ․ 2, p. 45-54.

Moreno, D. y Olmeda, I. (2003). Empleo de medidas de performance en la evaluación de fondos de inversión. En: Revista Estudios de la Bolsa de Madrid, n.․ 117, febrero, p. 58-62.

Pino, A.; Uribe, J. y Jiménez, D. (2017). Relevancia de los inversionistas institucionales en el mercado accionario colombiano. En: Semestre Económico, Vol. 20, n. ${ }^{\circ}$ 44, p. 45-66. DOI: 10.22395/seec.v20n44a3.

Sharpe, William. (1966). The Sharpe Ratio. En: Journal of Portfolio Management, Vol. 2, n. 1, p.63-75.

Treynor, J. (1966). How To Rate Management Of Investment Funds. En: Harvard Business Review, Vol. 43, n. ${ }^{\circ}$, septiembre, p. 63-75.

Uribe, J. y Fernández, J. (2014). Riesgo Sistémico en el mercado de acciones colombiano: altrernativas de diversificación bajo eventos extremos. En: Cuadernos de economía, Vol. 33, n. ${ }^{\circ}$ 63, febrero, p. 613-634.

Uribe, J. y Ulloa, I. (2011). Revisando la hipótesis del mercado eficiente; nuevos datos, nuevas crisis, nuevas estimaciones. En: Revista Cuadernos de Economía, Vol. 30, n. 55, septiembre, p. $127-154$. 
Estructuración de un portafolio de inversiones con acciones colombianas

Winston, W. (2011). Modelos Financieros con simulación y optimización. Indiana, Estados Unidos: Palisade Corporation, 487p.

Zhiwu, C., y Peter, K. (1996). Portfolios Measurement: Theory and Aplications. En: The Review of Financial Studies, Vol. 9, n. ${ }^{\circ}$ 2, april, p. 511-555. DOI: 10.1093/rfs/9.2.511. 